\title{
The DD Check App for prevention and control of digital dermatitis in dairy herds.
}

\author{
Marlène Tremblay ${ }^{1}$, Tom Bennett $^{1}$, Dörte Döpfer ${ }^{1}$ \\ "Departments of Medical Sciences, Section of Food Animal Production Medicine, School of Veterinary \\ Medicine, University of Wisconsin-Madison, 2015 Linden Drive, Madison 53706
}

\author{
Corresponding author: \\ Marlène Tremblay \\ University of Wisconsin-Madison \\ School of Veterinary Medicine \\ Department of Medical Sciences \\ Section of Food Animal Production Medicine
}

Office: 2002

2015 Linden Dr.

Madison, WI 53706

Phone: 608-263-1186

Email: mtremblay@wisc.edu 


\section{ABSTRACT}

Digital dermatitis (DD) is the most important infectious claw disease in the cattle industry causing outbreaks of lameness. The clinical course of disease can be classified using 5 clinical stages. M-stages represent not only different disease severities but also unique clinical characteristics and outcomes. Monitoring the proportions of cows per M-stage is needed to better understand and address DD and factors influencing risks of DD in a herd. Changes in the proportion of cows per M-stage over time or between groups may be attributed to differences in management, environment, or treatment and can have impact on the future claw health of the herd. Yet trends in claw health regarding DD are not intuitively noticed without statistical analysis of detailed records. Our specific aim was to develop a mobile application (app) for persons with less statistical training, experience or supporting programs that would standardize M-stage records, automate data analysis including trends of M-stages over time, the calculation of predictions and assignments of Cow Types (i.e., Cow Types I, II, III are assigned to cows without active lesions, single and repeated cases of active DD lesions, respectively). The predictions were the stationary distributions of transitions between DD states (i.e., M-stages or signs of chronicity) in a class-structured multi-state Markov chain population model commonly used to model endemic diseases. We hypothesized that the app can be used at different levels of record detail to discover significant trends in the prevalence of M-stages that help to make informed decisions to prevent and control DD on-farm. Four data sets were used to test the flexibility and value of the DD Check App. The app allows easy recording of M-stages in different environments and is flexible in terms of the users' goals and the level of detail used. Results show that this tool discovers trends in M-stage proportions, predicts potential outbreaks of DD, and makes comparisons among Cow Types, signs of chronicity, scorers or pens. The DD Check App also provides a list of cows that should be treated augmented by individual Cow Types to help guide treatment and determine prognoses. Producers can be proactive instead of reactive in controlling DD in a herd by using this app. The DD Check App serves as an example of how technology makes knowledge and advice of veterinary epidemiology widely available to monitor, control and prevent this complex disease. 


\section{INTRODUCTION}

Digital dermatitis (DD) is predominately caused by Treponema spp and is one of the most common causes of lameness in the dairy industry (Evans et al., 2008; Klitgaard et al., 2008; Yano et al., 2010; Gomez et al., 2012; Zinicola et al., 2015). In addition to issues of welfare, it also causes decreased profits partly due to involuntary culling, and decreased production and fertility (Amstel and Shearer, 2006; Cha et al., 2010). Research has shown that DD is multifactorial, polymicrobial disease that has a wide range of severities and clinical stages with different significance (Read et al., 1998). It affects individual cows differently, and disease can also differ among parities and stages of lactation (Palmer and O'Connell, 2015; Schöpke et al., 2015). Currently, the common treatment and control methods include topical treatment (either during routine hoof trims or initiated by severe lameness) and routine hoof baths for the entire herd. The approach in preventing and controlling DD is currently governed by a "one size fits all" attitude as if all cows reacted equally to interventions. Commonly, prevention and control measures are implemented as a reaction to an outbreak instead of a proactive strategy based on continuous monitoring of herd dynamics. Such proactive prevention and control strategies require in-depth investigations of DD lesions over time.

Similar to udder health, monitoring trends in DD prevalence at an individual cow level would lead to improved understanding and awareness of DD dynamics in the endemically affected herd. Currently, many cases of DD go unnoticed (Rodriguez-Lainz et al., 1996), because the focus of detection is on lame cows with active large DD lesions. This detection strategy misses many cases of DD, because not all DD affected cows are lame (Tadich, 2010). M-stages, M0-M4.1, have been used to describe the clinical course of DD including differences in severity of disease (Döpfer et al., 1997; Berry et al., 2012) and the M-stage system is reported to be a helpful tool for combatting DD (Schöpke et al., 2015). In addition to 
M-stages, signs of chronicity such as hyperkeratosis and proliferations of the skin affected by DD are important to record, as this also affects the resulting lameness, infectious potential, treatment needs and prognosis of the DD lesion (Gomez et al., 2014). Proactive and repeated record keeping of DD lesions and their signs of chronicity should be part of an integrated prevention and control strategy against DD that includes all ages of cattle, including the pre-calving heifers (Gomez et al., 2015).

Trends in M-stage prevalence over time at the herd level are related to changes in management (hygiene), environment (temperature), control and treatment (Rodriguez-Lainz et al., 1999; Wells et al., 1999; Somers et al., 2005; Holzhauer et al., 2006; Cramer et al., 2009). Predictions of such trends can be achieved at different levels of record detail, i.e., by M-stage records or just presence or absence records. The prediction tools needed are the stationary distributions of transitions between DD states (i.e., Mstages or signs of chronicity) using a class-structured population model (Otto and Day, 2007). Such a model would allow proactive prediction of outbreaks and effects of interventions before outbreaks and recurrence of DD happens in real time. The significance of such trends can be tested statistically using the uncertainty measures of the model outcomes (Chernick, 2008).

At the cow level, the recurrence of the active DD lesions (M2) allows for cows to be assigned so-called Cow Types (I, II, III). Cow Type is assigned based on history of M2 lesions (Döpfer et al., 2004; Holzhauer et al., 2006; Holzhauer et al., 2008; and Gomez et al., 2014). Cow Types have been shown to have different immunologic response to Treponema spp. (Gomez et al., 2014) and are therefore important for the prognosis of treatment and genetic selection against DD (Schöpke et al., 2015).

Although the mathematical and statistical tools exist that can achieve the predictions of DD states given history and trends over time, those tools are not available to everybody in the field. Our specific aim was to develop a mobile app to generate standardized DD records, assign Cow Types based on DD history, and to automate descriptive and predictive data analyses. This app would be available to users who have 
less statistical training background, experience or supporting programs. We hypothesized that the app can be used at different levels of record detail to discover statistically significant trends in the prevalence of M-stages that help make informed decisions to prevent and control DD on-farm. Given the complex nature of DD and the high prevalence of DD affected herds and cows, long-term prevention and control strategies against DD would benefit from automated description and prediction tools. Similar apps aimed at automating statistical analyses of standardized field data sets will increase the awareness and understanding by producers, managers, veterinarians and owners resulting in improved prevention and control of endemic production diseases.

\section{MATERIALS \& METHODS}

The iOS based Zinpro DD Check App is available through the Apple App Store (Apple Inc, Cupertino, CA, USA) or iTunes for the iPad or the iPhone. The app was developed in two parts: Mobile and Webbased portions. The mobile portion was developed using Xcode (http://developer.apple.com/tools/xcode/) and the web-based portion was developed using R Shiny version 3.1.2 (R Development Team, 2014).

\section{Mobile Portion}

The mobile portion has an interface for entering herd name, herd code, scorer, date, pen number, cow ID, foot, M-stage lesion, and signs of chronicity (see Figure 1A). The signs of chronicity are defined as: None: smooth skin without thickening, Hyperkeratotic: thickened skin, Proliferative: overly grown epidermal tissues.

To help users learn to correctly score DD lesions using the M-stage system, pictures and descriptions are supplied. The M-stages of DD are defined as: M0: normal digital skin, M1: an early, small circumscribed red to gray epithelial defect of less than $2 \mathrm{~cm}$ in diameter, M2: the acute, active ulcerative or granulomatous digital skin alteration greater than $2 \mathrm{~cm}$ in diameter, M3: the healing stage within 1 to $2 \mathrm{~d}$ 
after topical treatment, where the acute DD lesion has covered itself with a firm scab-like material, M4: the chronic stage of DD being either hyperkeratotic or proliferative, M4.1: a chronic stage with an early or intermediate stage (M1) on top of it (Berry et al., 2012, Evans et al., 2016). The iPad version uses a much larger screen and therefore demonstrates DD lesions on the scoring buttons. The interface supports accurate and rapid assessments of DD lesions. Cattle can be scored using all 5 M-stages (Berry et al., 2012), simplified M-stages where M3 and M4 are combined (Relun et al., 2011), just two M-stages to represent the absence or presence of DD (Winders et al., 2015), or any other combination that suits the user as long as consistency is present throughout the data set. If a cow had more than one lesion present during scoring at the cow level, M2 lesions were ranked over M3s, M3s were ranked over M4s, and M4 lesions were ranked over M1s. If more than one sign of chronicity was present, proliferation was ranked over hyperkeratosis. A scoring chart is provided in the appendix A (supplementary data).

Scoring can take place in the milking parlor (Relun et al., 2011), using a restraint chute, or during pen walks where cattle are in head locks at the feed aisle and the scorer walks behind the cattle scoring the hind feet (Jacobs et al., 2015). Free ranging cattle in freestalls can be scored while walking past the investigator one-by-one in the alleys. In addition, the app has been used to generate records in beef cattle operations while cattle are feeding at the feed bunk or using so-called DD alley checks. DD alley checks can be possible at any feedlot which has the necessary facilities (e.g., connecting alleys), and handlers with some experience and training. During DD alley checks cattle walk by the investigator in groups of 3 to 5 in a feedlot alley, stop at their pen gates for evaluations and return to their home pens.

Once a cow has been scored, the "Save/New Cow" button is pressed to save the data to an updated comma separated value file (.csv) and ready the input section for the next cow. The user can view the recorded data in the lower section of the app which can be selected by the current day, all days, all farms or just the M2 observations of a given day. The "Summary" button will provide the user with a listing of the individual records and summary with the number of records, percentages of feet scored and 
percentages of lesions scored. The app also enables the user to download and edit data. Finally, the saved data can be submitted to the second portion of the app using the "Submit data" button.

\section{Web-based Portion}

The second portion of the app is a web-based app that allows data to be submitted directly from the mobile app to a server in the cloud. The data is not stored on the server so the information is confidential. It supplies the user with the relative frequencies of M-stages or signs of chronicity per scoring event and predictions of future frequencies by applying the DD Infection Model. The web-based app's user interface has two sections: the left panel includes the user adjustments and the section on the right displays the results (see Figure 1B). The user interface slider can be used to select weeks of interest from the data set. The number of bootstraps that are used to make the predictions can be changed by the user depending on their needs. A dropdown menu of variables (i.e., herd name, herd code, pen, signs of chronicity, Cow Type, and scorer) is available to split the data into subsets to view their results separately.

Once the user has made the desired adjustments in the user adjustments panel and clicks the "Update View" button, the data are submitted for analysis. The automated data transformation begins by making an ID which is a combination of cow ID and foot if the specific foot was supplied. If foot was not supplied, records are identified at the cow level. Duplicate observations per week and IDs or empty cow IDs are removed. The dates are assigned to weeks and a Cow Type is assigned to each cow. Type I cows were never scored with an M2 lesion, Type II cows had one M2 lesion and Type III have repeat M2 lesions. Cow Type is assigned based on all records available and two records is the minimum number of records needed to define a Cow Type. Cow Type will always be assigned at the cow level even if records are detailed at the foot level. There is no maximum time limit between consecutive recordings to be able to assign a Cow Type. Consistent time intervals between frequent scoring events are ideal to increase the reliability, accuracy and interpretability of the assigned Cow Type. Finally a subset of the data will be created based on the weeks selected using the slider in the user interface. This data set with Cow Type 
added can be downloaded by means of a button "Download Cow Type Data" in the user input section (see Figure 1B). The final data set is then submitted to the DD Infection Model.

The DD Infection Model's predicted relative frequencies (i.e., predictions) of DD disease states (i.e., Mstages or signs of chronicity) are based on class-structured Multi-state Markov Chain Models (Otto and Day, 2007). Sequential scoring events are used to identify transitions among disease states. The total number of transitions between disease states per dataset is used to create a transition matrix. The transition matrixes are then converted into proportions. The predictions are the stationary distributions of the probability transition matrix, read from the first right eigenvector belonging to the dominant eigenvector (Otto and Day, 2007). The predictions' confidence intervals are generated using bootstrapping methods (Caswell, 2001). For every bootstrap integer selected in the user input section, the IDs are randomly sub-sampled with replacement to create a subset. Each subset is then passed through the DD Infection Model to produce predictions. The variance among all the subsets' predictions is used to generate the $95 \%$ confidence intervals of the predictions. Because bootstrapping has high computing times the initial number of bootstraps is set to 10 in the app. For the current analysis 1000 bootstraps were used. Increasing the number of bootstraps will increase computational time on average by 1.5 seconds per iteration depending on the size of the data set. There are no stringent rules for the number of bootstraps to be used for such models, but the higher the number of bootstraps the more reliable the $95 \%$ confidence intervals are (Henderson, 2005). Consistent time intervals between frequent scoring events are ideal to increase the accuracy of the predictions. To limit predictions made on low power data sets (e.g., small sample size, or too few cows being scored repeatedly) a limit of 15 transitions was placed before results would appear. Several errors messages were created to help users navigate most common mistakes or issues.

The results are supplied through graphs showing the observed relative frequencies of the disease states per week and future predictions. Each proportion includes $95 \%$ confidence intervals as error bars. The 
confidence intervals can be used as an indirect measure of significance among weeks or groups (Du Prel et al., 2009). Confidence intervals that do not overlap are an indication for a statistically significant difference in proportions. If the confidence intervals overlap, the difference in proportion is not statistically significant. The proportions and predictions are also available in table form under each graph.

There are 4 ways to examine results of the analysis (i.e., observed relative frequencies and predictions) which can be accessed by selecting different tabs in the web-based interface (see Figure 1B). The tabs are titled: Summary by M-stage, Summary by Chronicity, Graph by User Input Variable, Table of M2 Cows. The first tab, Summary by M-stage, supplies the results by M-stage. The second tab, Summary by Chronicity, supplies the results by sign of chronicity. If a variable was chosen in the dropdown menu located in the user adjustments, each subset created is passed through the DD Infection Model separately. These results can be attained in the third tab. The fourth tab supplies a list of cows that should be treated (i.e., cows with M2 lesions during the most recent scoring event) and includes each cow's previous Mstage and Cow Type for prognosis of topical treatment. Although M1, M2 and M4.1 lesions are all active lesions we choose to have the treatment list focus on M2 lesions because generally M1 and M4.1 lesions are not as painful as the M2 lesions (Holzhauer et al., 2008).

\section{Sample Data Sets}

Four data sets were used to demonstrate the key functions of the app. The data sets are described in Table 1.

Data set $A$ represents a longitudinal treatment and control field intervention trial comparing the effects of two types of foot bath solutions on a commercial dairy farm. The cows were selected from two comparable production pens which had been pre-screened for their starting M-stage prevalences weekly for the duration of 3 weeks. The cows were separated into two study groups that were matched for similar parities, days in milk, and DD Cow Types assigned using the first 3 scoring events. The cows were 
housed in 2 rows of freestalls at a $105-125 \%$ cow to freestall stocking ratio with sand bedding and no access to pasture. The control group (pen 3) walked through 2.6\% Copper Sulfate hoof baths once per day (after the evening milking), 5 days per week (Monday through Friday). The cows in the treatment group (pen 4) were directed to walk through an experimental foamed solution containing $2 \%$ glutaraldehyde and aluminum salts once a day (after the evening milking), five days per week (Monday through Friday) over the duration of the study. In both study groups cows walked through a footbath filled with water prior to entering the hoof bath. The pens were started on the treatment and control hoof bathing agents for two weeks before scoring events started. The hoof baths were emptied, cleaned, and replenished with a fresh hoof bath solution after approximately every 250-300 cows. Four scoring events (week 7-10), each one week apart, were performed by 6 investigators. Investigators were trained by one experienced person and re trained before every block of evaluations. Using a restraint chute, both hind feet of all cows were cleaned using disposable paper towels and scored using all 5 M-stages. During the entire study, acute M2 lesions were detected by the herdsmen once per day after morning milking and during weekly pen walks in between the scoring events. In addition, investigators detected and treated M2 lesions during scoring events. The acute M2 lesions were topically treated using topical oxytetracycline (Oxy-Mycin $100 \mathrm{mg} / \mathrm{mL}$; Agripharm Products, Westlake, TX) and a light wrap. Wraps were removed within 1 to 3 days. The final data set A consisted of 780 observations from 200 unique IDs at foot level. An additional scoring event occurred 3 weeks later (week 13). A total of 198 observations were collected during week 13 to compare the observed prevalences to the predictions made using data set A.

Data set $B$ consists of 1,959 observations from 1,058 cows scored on a commercial farm by one scorer using signs of chronicity and M-stages 0,2 , and 4 at the cow level. The scorer was a researcher with over 20 years of experience scoring DD M-stages. Cows were scored during 4 pen walks with average interval of 7.67 weeks (SD 1.53). Individual Cow Types were defined based on week 1 through week 24.

Data set $C$ consists of 7,144 observations at the cow level from 2,256 cows scored on a commercial farm. The same researcher as in data set B gathered these records during 5 pen walks with an average time interval of 11.25 weeks (SD 7.27) between scoring events. In order to record only the presence or absence 
of DD, cows were scored as either M0 or M4 as a placeholder for any DD lesion. In order to investigate the differences between heifers and older cows, the interface of Herd Code was used to enter if the cow was a heifer or cow by entering the number 1 or 2 , respectively.

Data set $D$ was gathered by a hoof trimmer on a commercial farm performing lesion scoring in a restraint chute. Six scoring events occurred at an average interval of 1.6 weeks (SD 0.89) between scoring events. The data set had 1,054 observations from 302 cows at the foot level (hind feet only) using signs of chronicity and M-stages $0,2,3$, and 4 .

\section{RESULTS}

\section{Data Set A}

The DD Check App allowed 780 observations of DD scores to be recorded more efficiently compared to manually recoding and transferring the data to an electronic file. Once the data were submitted to the web-based portion of the app, the variable Pen was selected as the user input variable to compare the treatment and control groups. Figure 2 illustrates the graphical outcome of the third tab (Graph by User Input Variable) using weeks 7 through 10 of Data Set A. Figure 2 also includes a bar for the proportions observed during week 13 to the right of the predictions. From week 7 to 10 , pen 3 had, on average, 53.8\% M0s, 25.6\% M1s, 5.2\% M2, 0.0\% M3, 1.3\% M4 and 14.2\% M4.1s. From week 7 to 10, pen 4 had, on average, $36.0 \%$ M0s, 32.1\% M1s, 5.9\% M2s, 0.5\% M3, 2.8\% M4 and 22.7\% M4.1s.

The prediction for the M2-stages based on weeks 7 through 10 for pen 3 was $1.2 \%(-0.3-0.03)$. The prediction for the M2-stages based on weeks 7 through 10 for pen 4 was 6.5\% (2.4 - 10.6). The predictions for the M2-stages based on weeks 7 through 10 are significantly different between pens (Figure 2), although the observed proportions of M2-lesion in the treatment group (pen 4) $5.1 \%$ (0.8 9.5) compared to the control group (pen 3) $2.0 \%(-0.8$ - 4.8) was not significantly different in week 10 (i.e., overlapping confidence intervals). Comparing the observed proportions from week 13, the observed 
proportions of M2-lesion in the treatment group compared to the control group became significantly different in week 13 as predicted (i.e., non-overlapping confidence intervals). In addition, the predicted proportions of M0 and M4.1 lesions were significantly different between pens but these significant differences did not materialize in the observed proportions of week 13 (see Figure 2).

In Table 2, the number of consecutive records per cow from weeks 7 through 10 are shown as transition matrices and probabilities scaled to 1 . For the control group (pen 3), 11.1\% (SD 7.8\%) of M2 lesions transitioned into M0s, while none of the M2 lesions in the treatment group (pen 4) transitioned into M0s. Very few transitions from and into the M3-stages were recorded, because M3 lesions are short-lived. M3 stages last for about 7 to 10 days before they develop into a healthy M0 stage, the chronic M4 or M4.1 or they develop into acute M2 stages (D. Döpfer, personal communication, 2016). In addition, records for M4 lesions were scarce while the M4.1 lesions were found much more frequently in comparison (see Table 2). The M4.1 lesions tended to stay M4.1 in more than 55\% of the cases for both groups. The M4.1 lesions in the treatment group though tended to progress more often into M2 compared to the control group.

\section{Data set B}

The DD Check App allowed 1,959 observations of DD scores to be recorded efficiently. Figures 3 and 4 illustrate the graphical outcomes of the "Summary by M-stage" and "Summary by Chronicity" tabs using Data Set B. On average, $19.8 \%$ of the cows had an M0, $19.3 \%$ had an M2, and $61.0 \%$ had an M4 lesion during each pen walk (see Figure 3). On average, $23.6 \%$ of cows did not show signs of chronicity, $28.5 \%$ had hyperkeratosis and $47.9 \%$ had proliferation per pen walk (see Figure 4).

The relative proportions of cows with M0 lesions were significantly higher in weeks 7, 16 and 24 compared to week 1 (Figure 3). The relative proportions of cows with M2 lesions were significantly 
higher in weeks 7, 16 and 24 compared to week 1 and the proportion of M4 were significantly lower in weeks 7,16 , and 24 compared to week 1 with the largest changes occurring between week 1 and 7 (Figure 3). The data set describes the trends in prevalences throughout the yearly period of increased risk (Berry et al., 2012). Week 1 represents late spring (May 21, 2014), week 7 represents summer (June 30, 2014), week 16 the early fall (September 04, 2014), and week 24 represents late fall (October 28, 2014).

The relative proportions of cows with no signs of chronicity were significantly higher in weeks 7 and 16 compared to week 1 and the proportion of lesions with hyperkeratosis were significantly lower in weeks 7, 16, and 24 compared to week 1 (Figure 4). The largest change in relative frequencies occurred between week 1 and 7. There was a significant decrease in M2 lesions between week 7 and 16 and cows with no sign of chronicity between weeks 7 and 16 and weeks 16 and 24. There was a significant increase in in M4 lesions between week 7 and 16. Transition matrixes and transition probabilities are available in the appendix B (supplementary data).

Tab 3 of the app describes significant differences found in the summary tabs by making subsets by Cow Type and by signs of chronicity. Figure 5 shows the outcome for each sign of chronicity separately. The significant rise in the relative proportion of M2 lesions and significant decrease in M4 lesions during week 7 seen in the summary tab (Figure 3) was also seen in the proliferative lesions subset (Figure 5). This increase in M2 lesions in week 7 was seen in the subset of scores without signs of chronicity although the differences were not significantly different. The hyperkeratosis subset did not show the trend for an increase in M2 lesions during week 7. Between weeks 16 and 24 there was a significant decrease in M2 lesions for the subset of scores without signs of chronicity and a significant increase in M2 lesions for the subset of scores with hyperkeratotic lesions.

Figure 6 shows the relative proportions and predictions for each Cow Type subset. Cow Type I and II both show a significant increase in M0 and a significant decrease in M4 lesions between week 1 and 7. 
Type II cows had a significant increase in M2 between week 1 and 7 and M4 between week 7 and 16. In addition, Type II cows had a significant decrease in M2 between weeks 7 and 16 and M4 lesions between weeks 1 and 7. Cow Type III cows had a significant increase in M2 between week 1 and 7. The increased proportion of Cow Type III cows with M2 lesions during week 7 reached 97.7\% during week 16. The relative proportion of M2 lesions was found to have a significant decrease between weeks 16 and 24 in the Cow Type III subset.

\section{Data Set C}

Data Set C had on average $65.2 \%$ of heifers with DD and $76.5 \%$ of cows with DD. The proportion of cows and heifers with DD were significantly different in 4 out of 5 scoring events (see Figure 7). There was a significant decrease in the proportion of cows with DD between week 1 and 23 and there was a significant increase in the proportion of heifers with DD in week 46 compared to week 38 . Predictions were also significantly different between cows and heifers. Transition matrixes and transition probabilities are available in the appendix B (Supplementary data).

\section{Data set D}

Data set D had on average 54.4\% M0s, 34.2\% M2, 9.8\% M3, and 1.7\% M4 lesions per scoring event (see Figure 8 ). On average $65.6 \%$ of cows in data set D had no signs of chronicity, $15.5 \%$ had hyperkeratosis and $18.9 \%$ had proliferation per scoring event (see Figure 9). Overall trends include an increase in the proportion of cows with M3 lesions and cows with proliferative lesions and a decrease in the proportion of cows with hyperkeratosis. The prediction for M0 lesions is significantly lower than the proportion seen in week 9. The predictions for M2 lesions and hyperkeratosis lesions are significantly higher than the proportion seen in week 9. Figure 10 shows the first several entries listed in the treatment list found under the fourth tab of the web-based app. Transition matrixes and transition probabilities are available in the appendix B (Supplementary data). 


\section{DISCUSSION}

Handheld devices such as the iPhone or iPad are becoming increasingly ubiquitous worldwide (World Health Organization, 2011). Apps available on these devices allow access to information and tools at all times. The ease of use make such applications an important part of the daily lives of many people especially those of younger generations. It is an ongoing process for dairy businesses to increasingly use this type of technology as part of their management decisions. Such applications aid producers in making improvements for their own herds and the standardized data sets generated help research about cattle husbandry and production. The current data analysis of data sets collected using the DD check App from a variety of farms is an example of automated data analysis for decision-making processes. The findings can be shared between farm health managers, and interpreted for statistical differences regarding trends, interventions and impact of control measures.

\section{Mobile part/interface}

The development of this app makes the task of tracking DD much more efficient. It removes the need to take records using pen and paper followed by manually transferring records to obtain an electronic file. Offering descriptions and sample photographs of different lesions helps maintain consistency among users and helps to train herd managers about the clinical course and aspects of DD. While maintaining consistency in the identification of M-stages, the mobile app interface still allows enough flexibility to be useful in different environments seen in the dairy industry. For example the app has been used to score cattle in restraint chutes, milking parlors, during pen walks and alley checks. The app has been used by a variety of investigators (e.g., hoof trimmer, managers, veterinarians and researchers) with different goals for using the app. Some users aim to use the DD Check App to examine data from a treatment and control study, to produce treatment lists, or to improve monitoring DD treatment and control effects on commercial or research farms. Although the ideal situation is to monitor DD at the M-stage level, the app is flexible enough to allow less experienced users to also monitor DD for just the absence or presence of 
the disease. If M-stages are used for recording DD, different levels of detail can be chosen depending on the user's preferences (e.g., all 5 M-stages, only 3 M-stages, inclusion of scores at the foot level etc). The interval between scoring events is also not fixed which allows users to pick what works best regarding their schedule and needs. Although the app's flexibility allows it to be used by a broad audience, it might limit how results can be compared among farms.

\section{Web portion}

Most producers have limited time or do not have the training to analyze data using statistical approaches. The web-based portion of the DD check app allows data from the mobile portion of the app to be summarized and distributed directly after collection. This increases users' motivation to invest the time to score cows and to follow DD trends. Depending on users' needs, the web-based results can be examined at different levels of detail. Monitoring trends in the relative frequencies of the M-stages is a feasible task for beginners while examining predictions, confidence intervals and separation by subsets might be better suited for more advanced users.

The transition between different lesions can take a few days, weeks, or up to several years (Döpfer et al., 1997; Berry et al., 2012; Krull et al., 2016). Although very frequent preferably weekly and consistent scoring events are preferable to avoid missing M-stage transitions between scoring dates, the short scoring intervals are not always feasible on farm. Our data sets' mean scoring intervals varied from 1 to 11.25 weeks. We suggest scoring intervals to be less than or equal to 1 month since greater intervals will increase the odds that two or more transitions will have taken place between evaluations (Berry et al., 2012). On endemically affected farms, this is of lesser consequence compared to farms suffering from acute outbreaks of disease. As intervals increase or become variable, the interpretation of the predictions should be undertaken with great caution and the observed relative frequencies should be evaluated instead. Note that the predictions are applicable at the average group level and may not describe the exact prediction for an individual animal. However, decisions about trends and outbreak prevention and control 
are taken at the population level and therefore using the model predictions represents a useful tool for decisions regarding claw health management. In addition, individual animals can be spotted for treatment and their diagnosis and prognosis can be taken into account using the M2 lesion treatment lists.

The precision for noticing significant differences among relative frequencies increases as sample size increases. Although significant differences between observed prevalences can be estimated by the overlap of confidence intervals, this ability to identify statistically significant differences in trends could be improved by adding automated statistical tests (e.g., chi-square test). In large herds it may not be feasible to score every cow on a regular basis. These farms can randomly select a proportion of the herd to be scored at regular time intervals to obtain an accurate representation about the impact of DD on the cows on a particular farm. The number of cows that need to be scored per farm results in a sample size that depends on a number of factors (e.g., lowest M-stage prevalence, the precision desired, total population size, the accuracy of the scorer, scoring technique) (Humphry et al., 2004; Thrusfield, 2005; Naing et al., 2006). It is important to minimize large changes in the population scored over time. Large changes in the population scored during different scoring events can artificially cause significant changes in proportions that are not representative of the herd level situation. In addition, not maintaining a consistent population to be scored decreases the amount of animals with consecutive scores contributing to the transition matrix and therefore, the predictions.

The app produces predictions of near future trends of DD in endemically affected farms. Although this could have many benefits, there are no guarantees that statistical predictions will manifest into reality and further research is required to validate the results. A class-structured multi-state Markov chain population model was chosen since the app was targeted towards herds endemically infected with DD. Constant transition rates between $\mathrm{M}$-stages over time imply an endemic state of the infection dynamics away from periods of outbreaks. Because outbreaks of infections tend to have non-linear features with increased transition rates due to explosive transmission of disease, the dynamic models of transmission such as the 
compartmental SIR models (Anderson and May, 1992) are more suitable for modeling outbreaks of infectious disease. In addition, a transmission model of infection such as the one reported by Döpfer et al. (2012) applies to the outbreak situation of DD. Future models that are driven by clinical hypothesis about meaningful transitions could model M-stages and signs of chronicity in the same model and include a selection of transitions that make exclusively clinical sense (for example: it does not make clinical sense for a healthy foot to make a transition into M2 without passing through other stages first). Sojourn times (the time units spend in the different clinical stages of disease) and covariates such as lactation number or production levels could complement the outcomes and interpretation of the transition analysis (Caswell, 2001).

\section{Data set A}

Data set A was collected during a treatment and control field trial and demonstrated the application of the DD Check App in a research setting using numerous repeated scoring events and all $5 \mathrm{M}$-stages while cows were scored in a stand-up restraint chute. The significant differences among observed and predicted prevalences of M-stages between the treatment and control pens support that the $2.6 \%$ Copper Sulfate hoof baths performed significantly better in controlling DD compared to the test footbath agent in the treatment group.

The transition analysis can be used to study the precursors to more severe lesions such as the M2-stages. This information can be used to revisit and intensify the monitoring and topical treatment strategies for individual cows and groups of cows as part of an informed decision making process. For example, if the results of the transition analysis had been available at the end of week 10 in the case of Data set A, the fact that the M2-stage prevalences between intervention groups were developing into statistically different prevalences could have been predicted before the week 13 scoring event. At the same time, the M4.1 stages could have been identified as the main pre-cursors to those M2-lesions in the treatment pen (Table

2). These results have major implications for the prevention and control of DD. The M4.1 lesions tended 
to stay M4.1 in more than 55\% of the cases in both pens. This could reflect the lesions resilience to the effects of the hoof bathing protocol. In addition, the signs of chronicity of these lesions and the effect of the hoof baths at keeping the M4.1 lesions stable and preventing them from progressing into M2 lesions are reflected and predicted by the results of the prediction model. The M4.1 lesions in the treatment group though tended to progress more often into M2 and M4s compared to the control group. The transition rates are probably not constant as the decrease in prevalence of M4.1 lesions in the treatment group on evaluation day 13 was significantly lower than predicted by the DD Infection Model. In addition, the difference between the predictions and the week 13 observed proportions could be due to the change from 1 week scoring intervals to a 3 week interval between weeks 10 and 13 . If the prediction model results would have been available at the time the manager could have intensified the hoof bathing frequency to try to prevent the significant difference in M2 lesions discovered in week 13. In addition, the systematic search for M2 lesions in the pens could have been intensified in order to detect these lesions earlier and to treat them promptly.

\section{Data set B}

Data set B was collected on a commercial dairy farm with relatively large intervals between scoring events (average 7.67 weeks) and a low percent of cows had repeat scores (35.5\%, see Table 1). Even though the data set used large scoring intervals, the trends in the observed relative frequencies revealed a significant increase in the proportion of cows without DD (M0) or signs of chronicity during weeks 7 and 16. There was also a significant rise in M2 lesions during the summer (week 7). This pattern of increased acute DD during the summer has been seen consistently found in the industry (Berry et al., 2012). There are many possible risk factors associated with this time of year such as temperature, management changes (lack of detection or treatment) or production factors. When broken down by signs of chronicity and Cow Type subsets the increase in M2 lesions during the summer can be further characterized as being associated with Cow Type II and III cows with proliferative lesions. Using these findings, the farm could be advised to intensify monitoring during late spring in order to treat new M2 lesions and prevent the 
increase of proliferations by customizing frequencies of hoof baths. This should help prevent the summer rise in acute DD.

\section{Data set C}

For producers that use the DD Check App on one farm only, the herd code or herd name interface can be used to record other variables of interest. Data set $\mathrm{C}$ illustrated that this could be used to record cow and heifer records separately. The records were then used to compare results of absence or presence of DD based on these subsets. This can also be done by making subsets of the comma separated value file manually, then submitting them to the web interface of the app separately, and comparing results ${ }^{1}$. Other possible variables of interest for analyzing differences between strata are heifer origin (different heifer growers), different environments on the same farms such as different barns, and scoring type such as milking parlor, pen walk, alley checks or chute evaluations. The results of data set $\mathrm{C}$ show that heifers have lower prevalence of DD for weeks 1 through 38 compared to cows. The confidence intervals for the prevalence of DD in cows and heifers overlap in week 46 which coincided with a change in management resulting in the lack of hoof bathing between week 38 and 46. Since that change in management, footbaths were no longer changed frequently enough and new lesions were not topically treated with topical antibiotics and wraps. This resulted in a significant increase of DD prevalence in heifers although a similar change in cows was not seen. The app made this change evident where it would have otherwise gone unnoticed. After discussing the findings from the DD Check App, the manager became more motivated to address the decline in DD treatment, prevention and control procedures.

\section{Data set D}

Data set $\mathrm{D}$ was gathered by a hoof trimmer using the treatment list located in the fourth tab of the webbased app. The treatment list supplies a cow's Cow Type that helps with prognosis and decisions such as

\footnotetext{
${ }^{1}$ Please contact Zinpro Corporation representatives at zinpro.com for the link to the web interface and login information
} 
breeding and culling. It is to be expected based on clinical expertise that cows with repeated M2 lesions have worse prognoses than cows without repeated M2 lesions. This data set also illustrated that future predictions of M-stage proportions can help to support the need to intervene or to continue with a preventive hoof bathing protocol by showing significant effects (e.g., rise in the proportion of M2 lesion and hyperkeratotic lesions) earlier than by using observational data alone. In this case, predictions helped to alert the hoof trimmer and herd manager regarding a potential outbreak and to draw attention to an increase in signs of chronicity before they occurred. The prediction tool allowed them to justify the increase of topical treatment efforts.

\section{CONCLUSION}

The development of the DD Check App successfully standardizes the recording of M-stages of DD, and the app automated the descriptive and predictive analyses of such longitudinal data. The app is flexible enough to be used at different levels of record detail and in different situations to discover statistically significant trends in the observed or predicted prevalence of M-stages to help make informed decisions to prevent and control DD on-farm. The app assigns Cow Types, creates treatment lists, and produces data sets for distribution between herd managers. It can be utilized by users who do not have a statistical background to predict near future trends of DD in endemically affected farms. Cattle can be scored during pen walks, at the milking parlor or using a cattle chute, and the app can be used to compare different subsets of cows. Similar apps aimed at standardizing field data sets will increase the awareness and understanding in the industry resulting in improved prevention and control of endemic production diseases. Future applications will include economic models to estimate cost and return of treating certain DD lesions or implementing prevention and control methods. Prevalence and cow characteristics from farm management programs could be linked to the model to visualize the association of cow and farm level risk factors with DD. 


\section{CONFLICTS OF INTEREST: none}

\section{ACKNOWLEDGEMENTS}

We would like to acknowledge Alison F. Dusick (University of Tennessee), Nigel Cook (UW-Madison), and Jane Rieman (UW-Madison) for their contributions and Mike Socha, Jeff Wheeler, Dana Tomlinson, Brent Wilson and Arturo Gomez from Zinpro Corporation for their support. We would like to thank the student hourly researchers: Nella Bernardoni, Kelly Pertzborn, Chavalin Chadwick, and Kristin Kerrish and hoof trimmers: Jamie Sullivan, Karl Burgi, Rick Trinko, Josh Potwell, Jason Wiedmeyer, and Nathan Henson for their help. We are grateful to the owners and employees of the participating dairy farms for their time and patience and to all the students at UW-Madison that contributed on a temporary basis.

This study was supported by Zinpro Corporation (Eden Prairie, MN, USA), and Ecolab Inc. (Redmond,

WA, USA). The project was funded in part by USDA-Animal Health R\&D Program Grant \#wis01417. The work of Alison F. Dusick was supported by NIH \#T32 RR17503.

\section{REFERENCES}

Amstel, S., Shearer, J., 2006. Manual for treatment and control of lameness in cattle. Blackwell Publishing, Iowa, USA, pp. 3-4.

Anderson, R.M., May, R.M., 1998. Infectious Diseases of Humans: Dynamics and Control. Oxford University Press, New York., pp. 122-126.

Berry, S.L., Read, D.H., Famula, T.R., Mongini, A., Döpfer, D., 2012. Long-term observations on the dynamics of bovine digital dermatitis lesions on a California dairy after topical treatment with lincomycin HCl. Vet. J. 193, 654-658. 
Caswell, H., 2001. Matrix population models : Construction, analysis and interpretation. 2nd ed. Sinauer Associates, Sunderland, Massachusetts.

Cha, E., Hertl, J.A., Bar, D., Gröhn, Y.T., 2010. The cost of different types of lameness in dairy cows calculated by dynamic programming. Prev. Vet. Med. 97, 1-8.

Chernick, M.R., 2008. Bootstrap methods: A guide for practitioners and researchers. 2nd edn. John Wiley \& Sons, Inc., Hoboken, New Jersey, 174 pp.

Cramer, G., Lissemore, K.D., Guard, C.L., Leslie, K.E., Kelton, D.F., 2009. Herd-level risk factors for seven different foot lesions in Ontario Holstein cattle housed in tie stalls or free stalls. J. Dairy Sci. 92, 1404-1411.

Döpfer, D., Koopmans, A., Meijer, F.A., Szakall, I., Schukken, Y.H., Klee, W., Bosma, R.B., Cornelissevan, J.L., Asten, A.J., ter Huurne, A.A.H.M. 1997. Histological and bacteriological evaluation of digital dermatitis in cattle, with special reference to spirochaetes and Campylobacter faecalis. Vet. Rec. 140, 620-623.

Döpfer, D., van Boven, R.M., de Jong, M.C.M., 2004. A mathematical model for the dynamics of digital dermatitis in dairy cattle. In Proceedings of the 13th International Conference for Production Disease, 1-5 October 1984, Lansing, MI, USA, pp. 36.

Döpfer, D., Holzhauer, M., van Boven, M., 2012. The dynamics of digital dermatitis in populations of dairy cattle: Model-based estimates of transition rates and implications for control. Vet. J. 193, $648-653$.

Du Prel, J.B., Hommel, G., Röhrig, B., Blettner, M., 2009. Confidence Interval or P-Value?: Part 4 of a Series on Evaluation of Scientific Publications. Deutsches Ärzteblatt International 106, 335-339.

Evans, N.J., Brown, J.M., Demirkan, I., Murray, R.D., Vink, W.D., Blowey, R.W., Hart, C.A., Carter, S.D., 2008. Three unique groups of spirochetes isolated from digital dermatitis lesions in UK cattle. Vet. Microbial. 130,141-150.

Evans, N.J., Murray, R.D., Carter, S.D., 2016. Bovine digital dermatitis: Current concepts from laboratory to farm. Vet. J. 211, 3-13. 
Gomez, A., Cook, N.B., Bernardoni, N.D., Rieman, J., Dusick, A.F., Hartshorn, R., Socha, M.T., Read, D.H., Döpfer, D., 2012. An experimental infection model to induce digital dermatitis infection in cattle. J. Dairy Sci. 95, 1821-1830.

Gomez, A., Anklam, K. S., Cook, N. B., Rieman, J., Dunbar, K. A., Cooley, K. E., Socha, M.T., Döpfer, D., 2014. Immune response against Treponema spp. and ELISA detection of digital dermatitis. J. Dairy Sci. 97, 4864-4875.

Gomez, A., Cook, N. B., Socha, M. T., Döpfer, D., 2015. First-lactation performance in cows affected by digital dermatitis during the rearing period. J. Dairy Sci. 98, 4487-4498.

Henderson, A.R., 2005. The bootstrap: a technique for data-driven statistics. Using computer-intensive analyses to explore experimental data.Clinica Chimica Acta 359, 1-26.

Holzhauer, M., Hardenberg, C., Bartels, C.J.M., Frankena, K., 2006. Herd-and cow-level prevalence of digital dermatitis in the Netherlands and associated risk factors. J. Dairy Sci. 89, 580-588.

Holzhauer, M., Bartels, C.J.M., Döpfer, D., van Schaik, G., 2008. Clinical course of digital dermatitis lesions in an endemically infected herd without preventive herd strategies. Vet. J. 177, 222-230.

Humphry, R. W., Cameron, A., Gunn, G. J., 2004. A practical approach to calculate sample size for herd prevalence surveys. Prev. Vet. Med. 65, 173-188.

Jacobs, C., Orsel, K., Morabito, E., Barkema, H. M., 2015. Pen walks as a tool to determine the prevalence of digital dermatitis in young stock on Alberta dairy farms. Proc. Of 18th International Symposium/10th International Conference on Lameness in Ruminants, Valdivia, Chile, pp. 129.

Klitgaard, K., Boye, M., Capion, N., Jensen, T.K., 2008. Evidence of multiple Treponema phylotypes involved in bovine digital dermatitis as shown by $16 \mathrm{~S}$ rRNA gene analysis and fluorescence in situ hybridization. J. Clin. Microbiol. 46, 3012-3020.

Krull, A.C., Shearer, J.K., Gorden, P.J., Scott, H.M., Plummer, P.J., 2016. Digital dermatitis: Natural lesion progression and regression in Holstein dairy cattle over 3 years. J. Dairy Sci. 99, 37183731. 
Naing, L., Winn, T., Rusli, B.N., 2006. Practical Issues in Calculating the Sample Size for Prevalence Studies. Archives of Orofacial Sciences 1, 9-14.

Otto, S.P. and Day, T., 2007. A biologist's guide to mathematical modeling in ecology and evolution. Vol. 13. Princeton University Press, Princeton, pp. 396-398.

Palmer, M.A., O'Connell, N.E., 2015. Digital Dermatitis in Dairy Cows: a review of risk factors and potential sources of between-animal variation in susceptibility. Animals 5, 512-535.

R Development Core Team, 2014. R: A Language and Environment for Statistical Computing. R Foundation for Statistical Computing, Vienna, Austria, ISBN 3-900051-07-0, URL http://www.R-project.org/

Read, D.H., Walker, R.L., 1998. Papillomatous digital dermatitis (footwarts) in California dairy cattle: clinical and gross pathologic findings. J.Vet. Diagn. Invest. 10, 67-76.

Relun, A., Guatteo, R., Roussel, P., Bareille, N., 2011. A simple method to score digital dermatitis in dairy cows in the milking parlor. J. Dairy Sci. 94, 5424-5434.

Rodriguez-Lainz, A., Melendez-Retamal, P., Hird, D.W. 1996. Prevalence of papillomatous digital dermatitis in dairy herds in Chile. Proc. of 77th Annual Meeting of Conference of Research Workers in Animal Diseases (abstract), Chicago, USA. No. 46.

Rodriguez-Lainz, A., Melendez-Retamal, P., Hird, D.W., Read, D.H. and Walker, R.L., 1999. Farm-and host-level risk factors for papillomatous digital dermatitis in Chilean dairy cattle. Prev. Vet. Med. 42, 87-97.

Schöpke, K., Gomez, A., Dunbar, K.A., Swalve, H.H, Döpfer, D., 2015. Investigating the genetic background of bovine digital dermatitis using improved definitions of clinical status. J. Dairy Sci. $98,8164-8174$.

Somers, J.G.C.J., Frankena, K., Noordhuizen-Stassen, E.N., Metz, J.H.M., 2005. Risk factors for digital dermatitis in dairy cows kept in cubicle houses in The Netherlands. Prev. Vet. Med. 71, 11-21.

Tadich, N., Flor, E., Green, L., 2010. Associations between hoof lesions and locomotion score in 1098 unsound dairy cows. Vet. J. 184, 60-65. 
Thrusfield, M., 2005. Veterinary Epidemiology, 3rd ed. Blackwell Science Limited, Oxford, UK., pp. 233-234.

Wells, S.J., Garber, L.P., Wagner, B.A., 1999. Papillomatous digital dermatitis and associated risk factors in US dairy herds. Prev. Vet. Med. 38, 11-24.

Winders, T., Socha, M., Cramer, G., 2015. An evaluation of the agreement between digital dermatitis scoring methods in the parlor, pen and hoof-trimming chute. Proc. Of 18th International Symposium/10th International Conference on Lameness in Ruminants, Valdivia, Chile, pp. 134.

World Health Organization, 2011. mHealth: New Horizons for Health Through Mobile Technologies: Second Global Survey on eHealth. Global Observatory for eHealth Series, 3. Geneva: WHO, 2011.

Yano, T., Moe, K.K., Yamazaki, K., Ooka, T., Hayashi, T., Misawa, N., 2010. Identification of candidate pathogens of papillomatous digital dermatitis in dairy cattle from quantitative $16 \mathrm{~S}$ rRNA clonal analysis. Vet. Microbiol. 143, 352-362.

Zinicola, M., Lima, F., Lima, S., Machado, V., Gomez, M., Döpfer, D., Guard, C., Bicalho, R., 2015. Altered Microbiomes in Bovine Digital Dermatitis Lesions, and the Gut as a Pathogen Reservoir. PloS one 10, e0120504. 
Figure 1: Screen shots of the DD Check App user interface: A) mobile portion- data entering screen; B) web portion- screen where data is analyzed

Figure 2: Output of the third tab (Graph by User Input Variable) separated by pen showing the relative proportions of hind feet in each M-stage by week and predictions based on weeks 7 through 10 of Data Set A. The observed proportions from week 13 were added as the bar to the right of the lighter gray bar with the predictions for comparisons; Notes: Pred. - Prediction; Error bars- 95\% confidence intervals using 1000 bootstraps, $n=100$ cows and 200 unique feet records resulting in a total of 978 observations

Figure 3: Output of the first tab (Summary by M-stage) for Data Set B showing the relative proportions of cattle in each M-stage by week and predictions. Notes: Error bars - 95\% confidence intervals using 1000 bootstraps; Pred. - Prediction

Figure 4: Output of the second tab (Summary by Chronicity) for Data Set B showing the relative proportions of cattle in each sign of chronicity by week and predictions. Notes: Error bars - 95\% confidence intervals using 1000 bootstraps; Pred. - Prediction

Figure 5: Data Set B - Output of the third tab (Graph by User Input Variable) separated by sign of chronicity showing the relative proportions of cattle in each M-stage by week and predictions. Notes: Error bars - 95\% confidence intervals using 1000 bootstraps; Pred. - Prediction

Figure 6: Data Set B- Output of the third tab (Graph by User Input Variable) separated by Cow Type showing the relative proportions of cattle in each M-stage by week and predictions. Notes: Error bars 95\% confidence intervals using 1000 bootstraps; Pred. - Prediction 
Figure 7: Data Set C- Output of the third tab (Graph by User Input Variable) separated by Herd Code which was used to differentiate parity group (cows from heifers). The figure shows the relative proportions of cattle in each M-stage per parity group by week and predictions. Notes: Error bars - 95\% confidence intervals using 1000 bootstraps; Pred. - Prediction

Figure 8: Output of the first tab (Summary by M-stage) for Data Set D showing the relative proportions of hind feet in each M-stage by week and predictions. Notes: Error bars - 95\% confidence intervals using 1000 bootstraps; Pred. - Prediction

Figure 9: Output of the second tab (Summary by Chronicity) for Data Set D showing the relative proportions of hind feet in each sign of chronicity by week and predictions. Notes: Error bars - 95\% confidence intervals using 1000 bootstraps; Pred. - Prediction

Figure 10: Sample of the output of the fourth tab (Table of M2 Cows) for Data Set D 


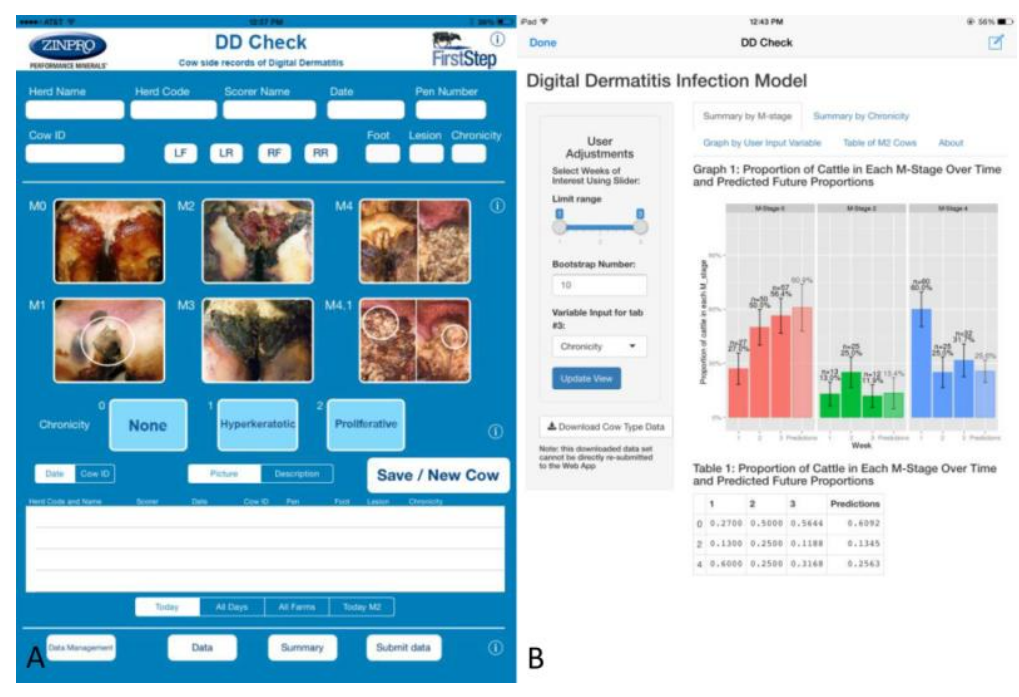




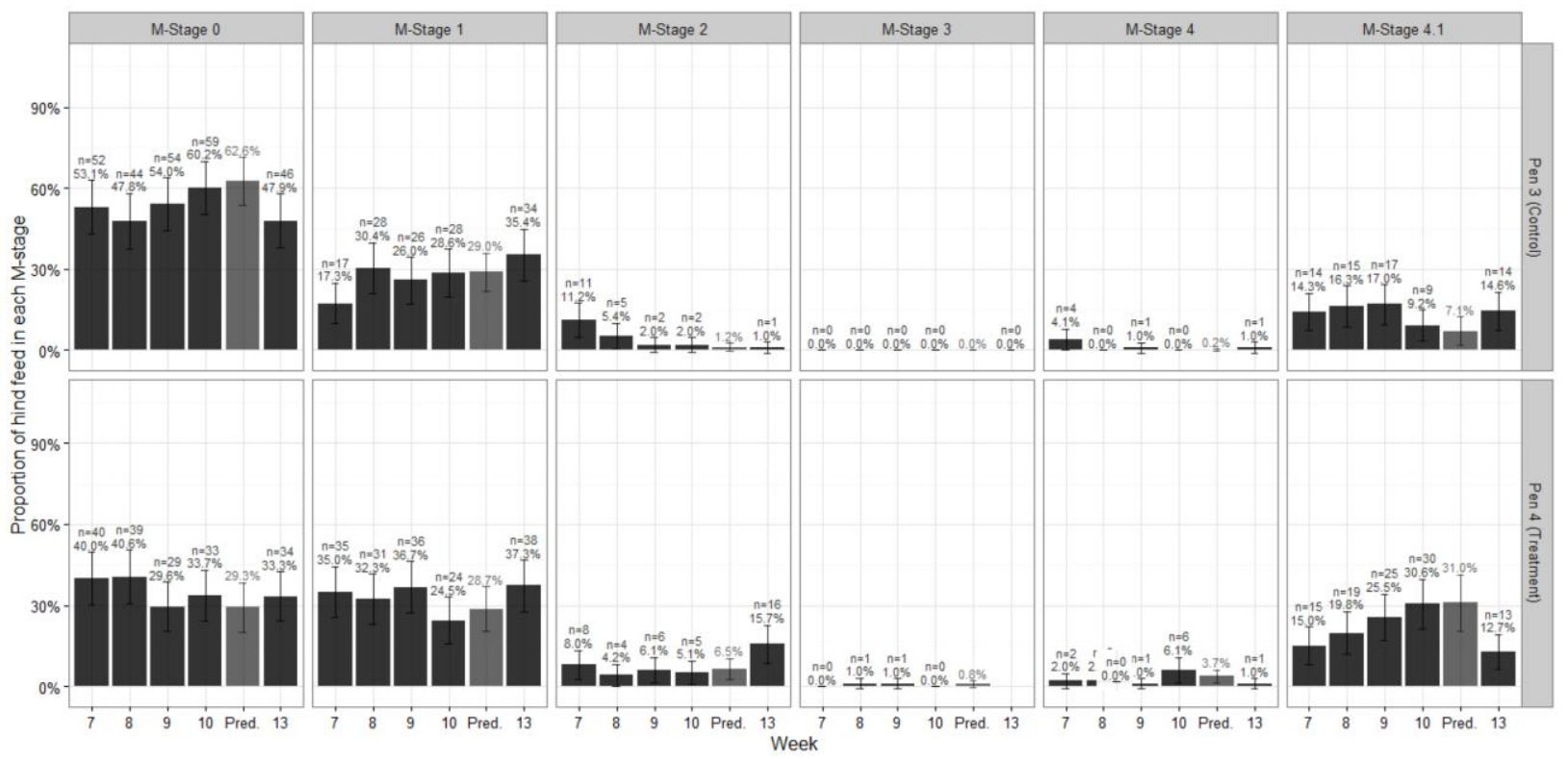




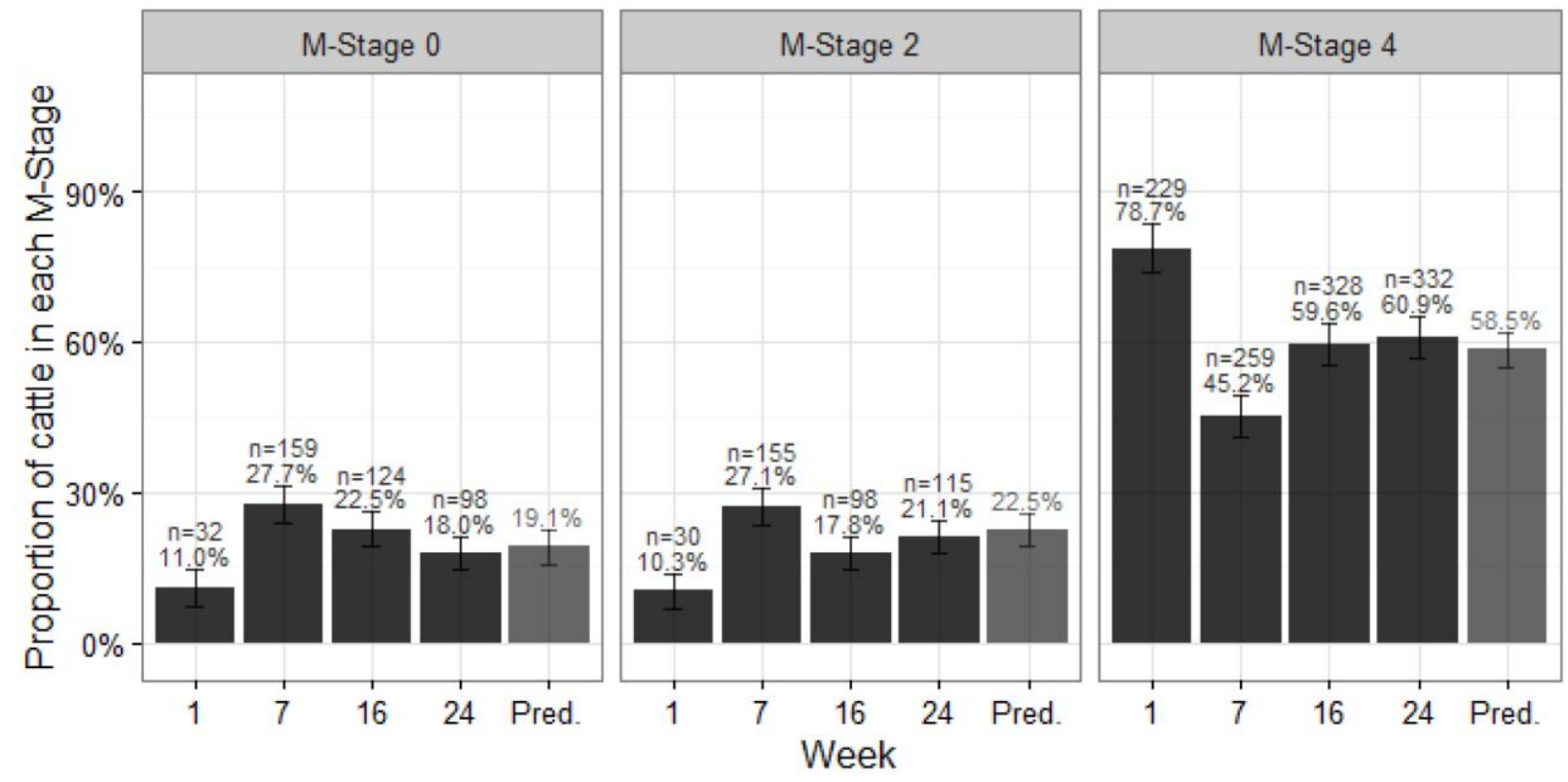




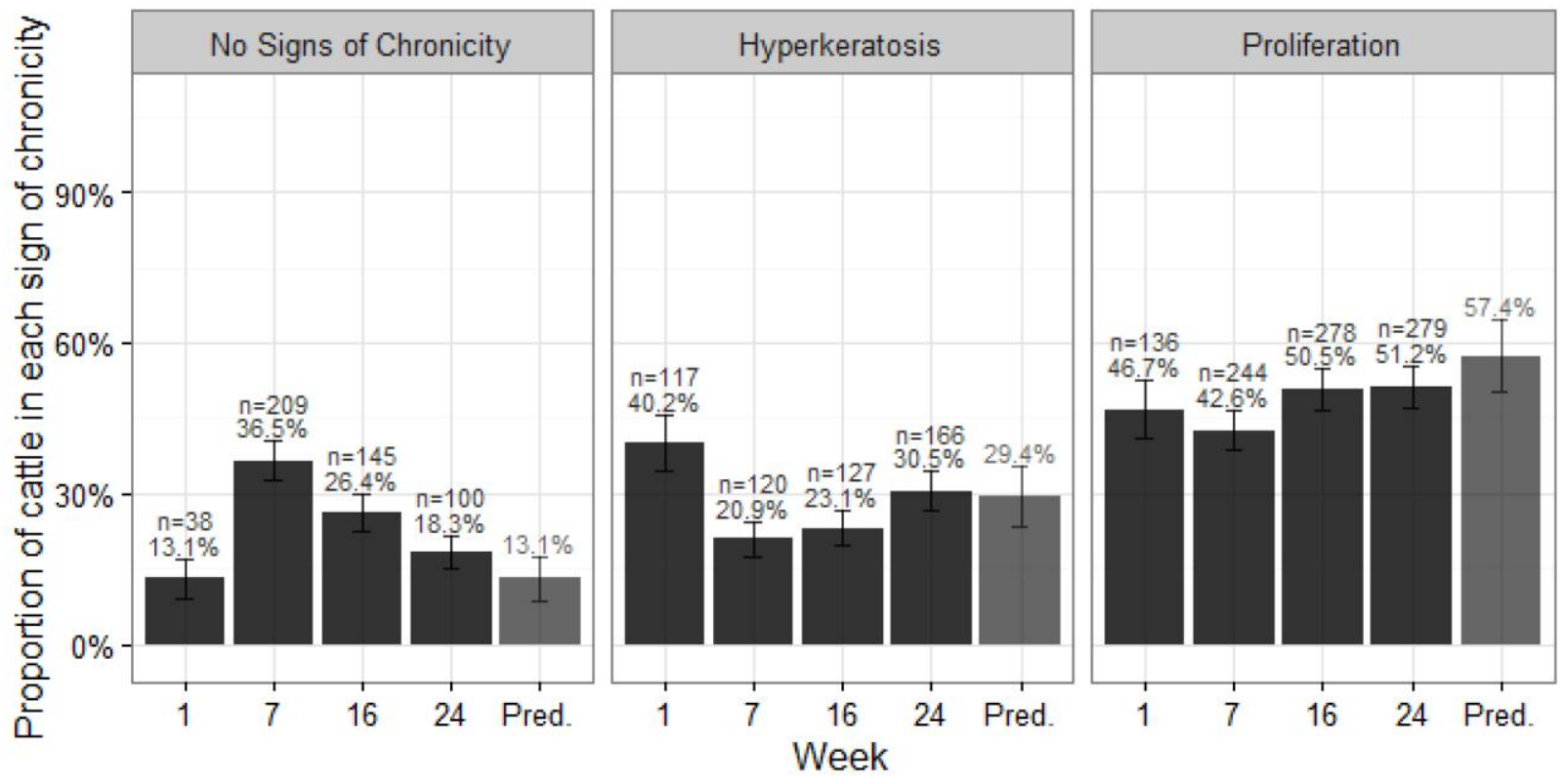




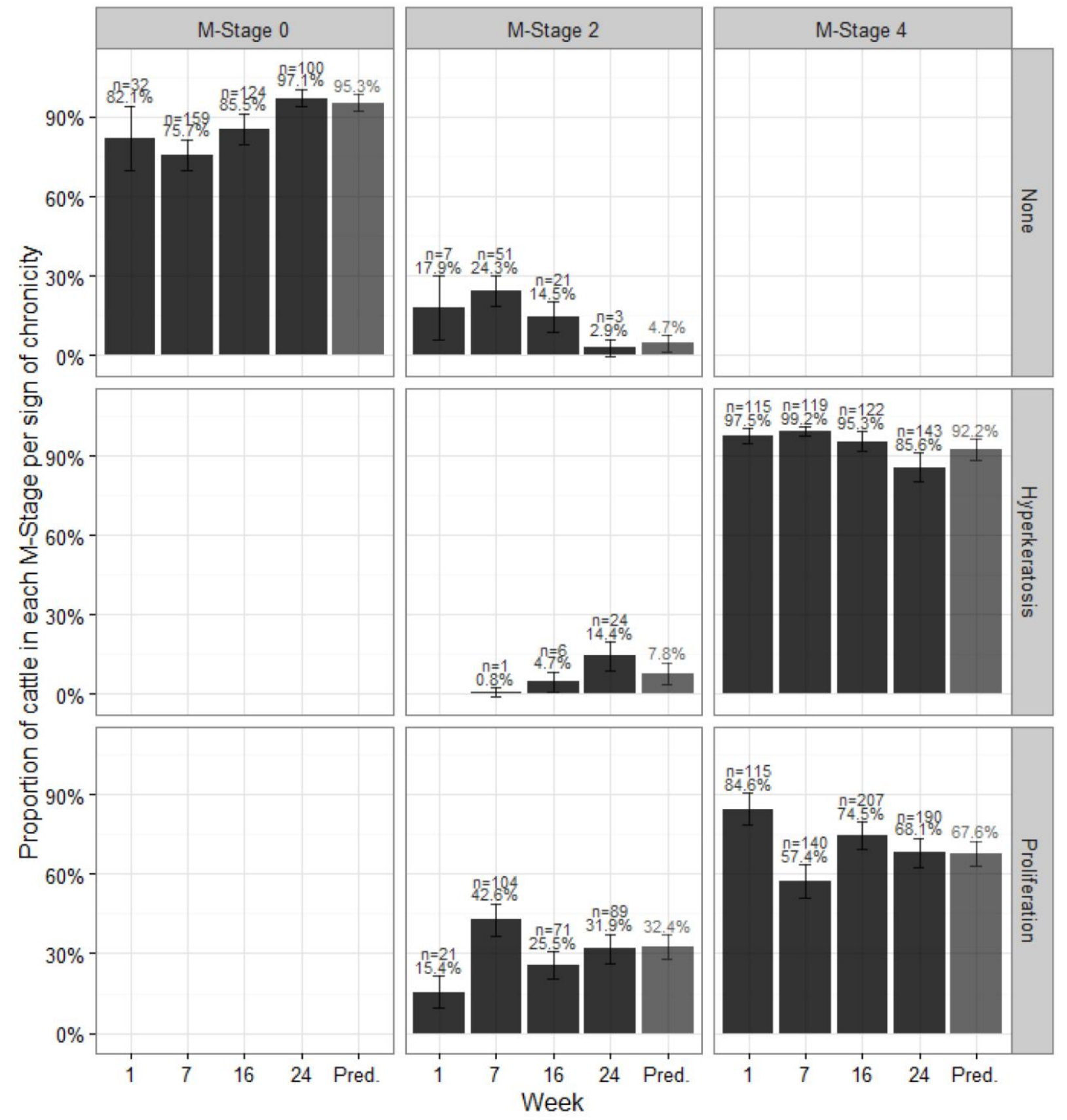



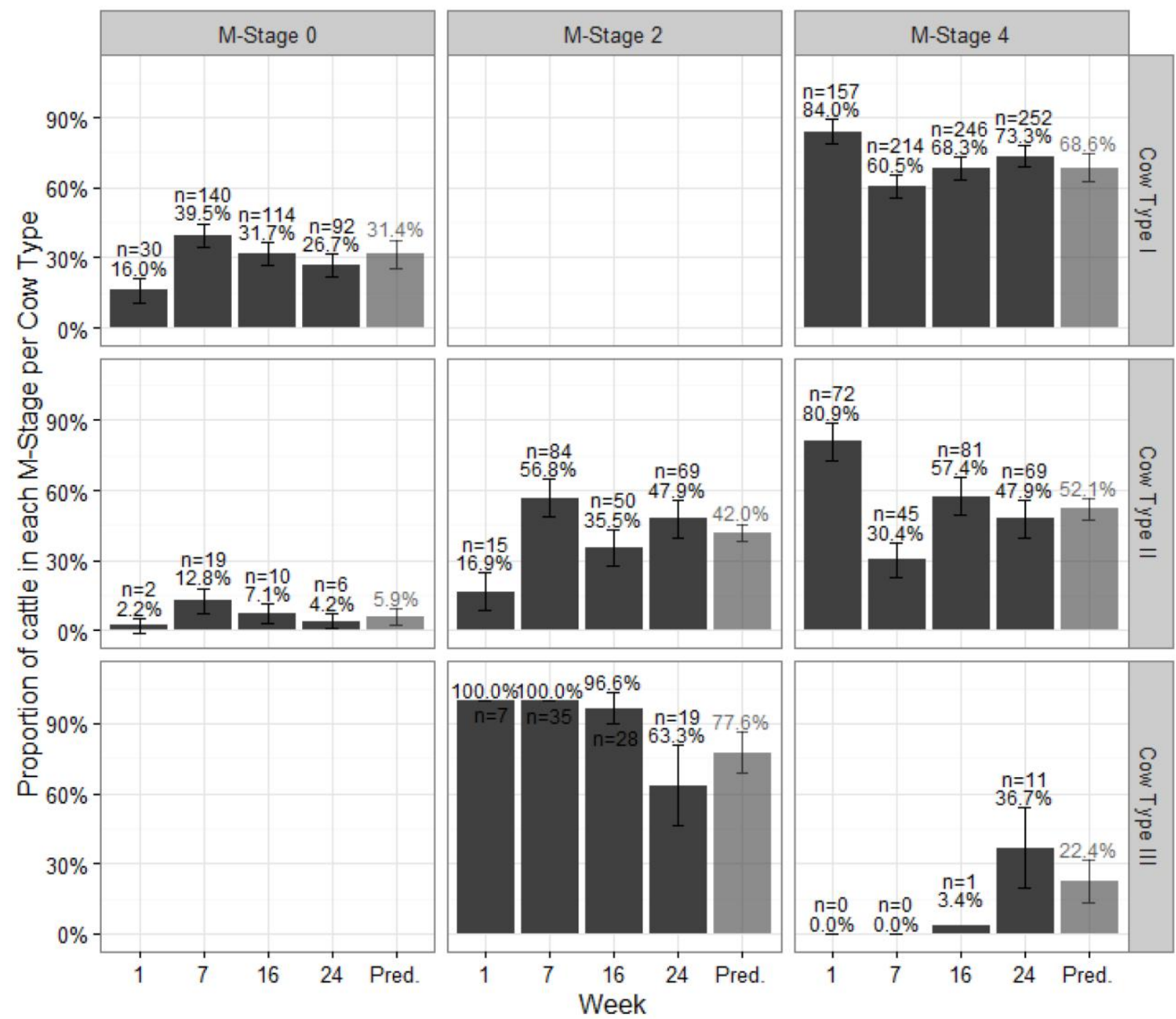


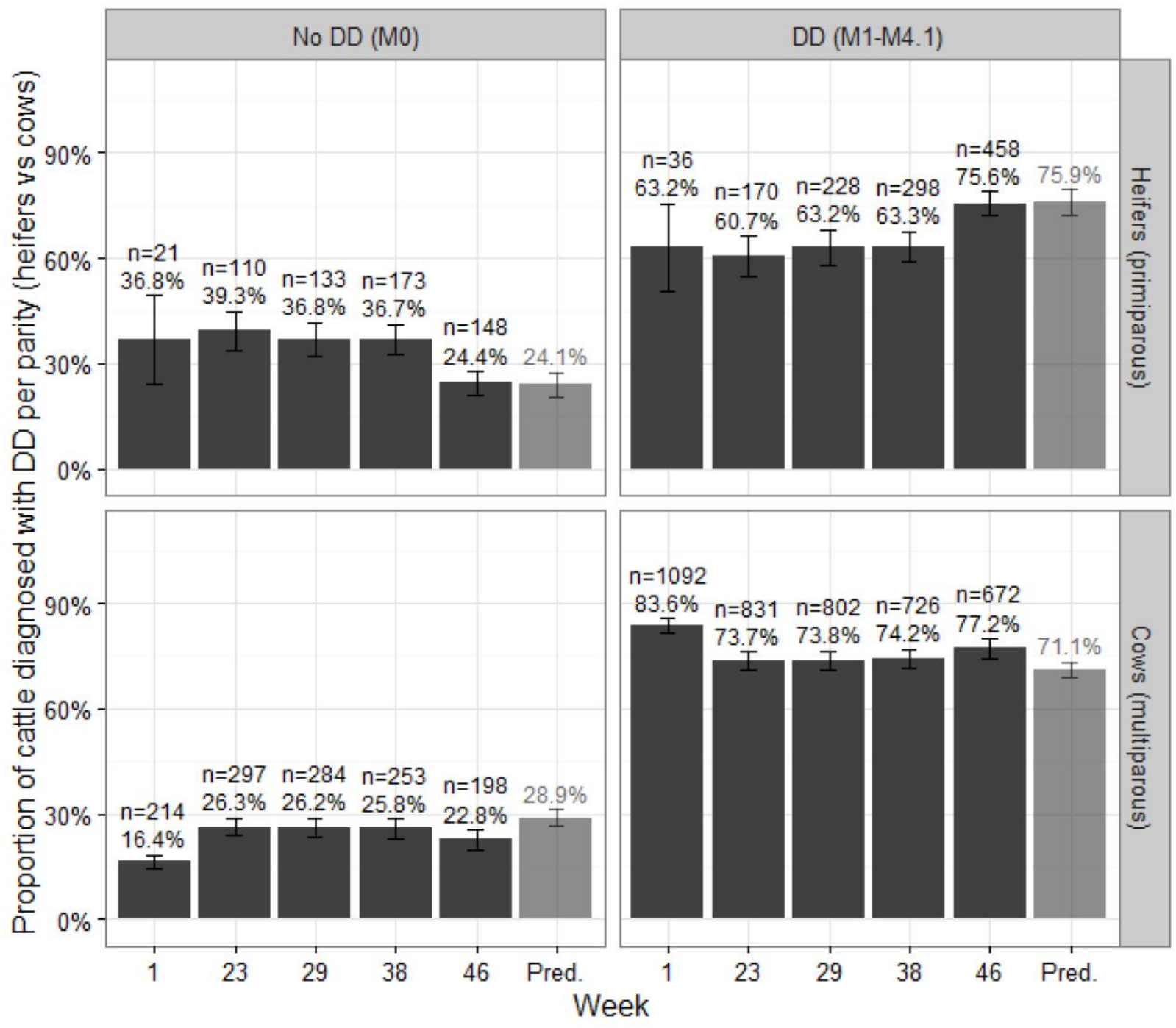




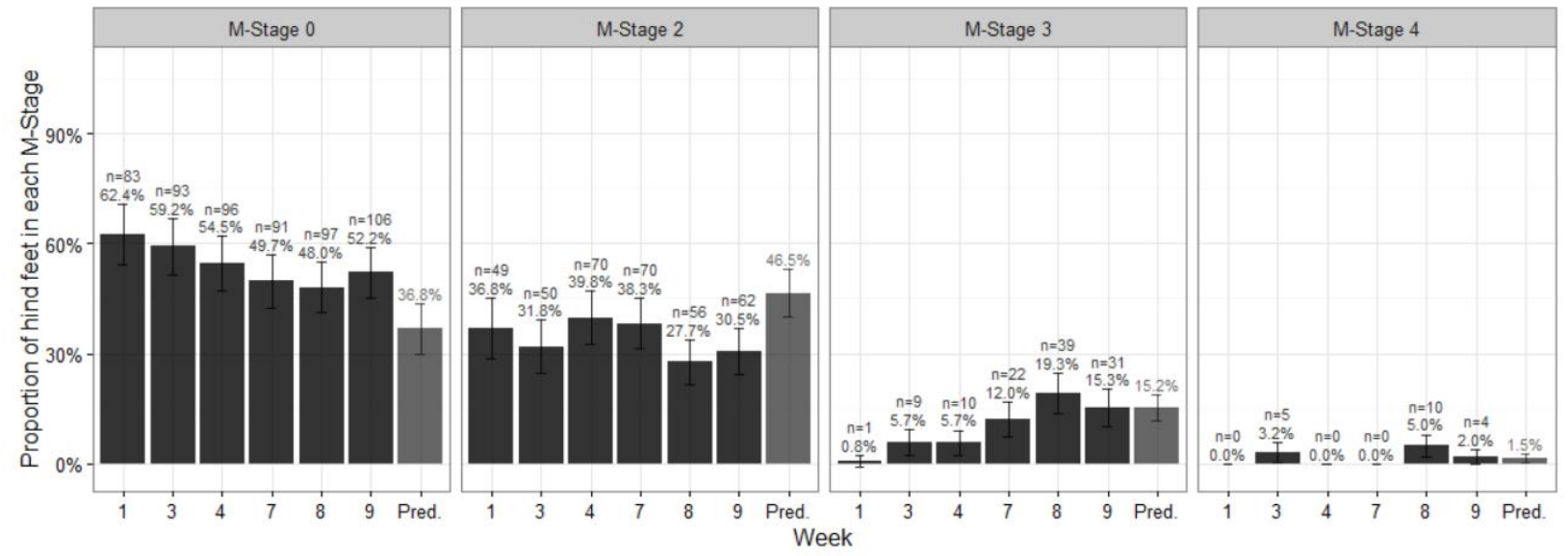




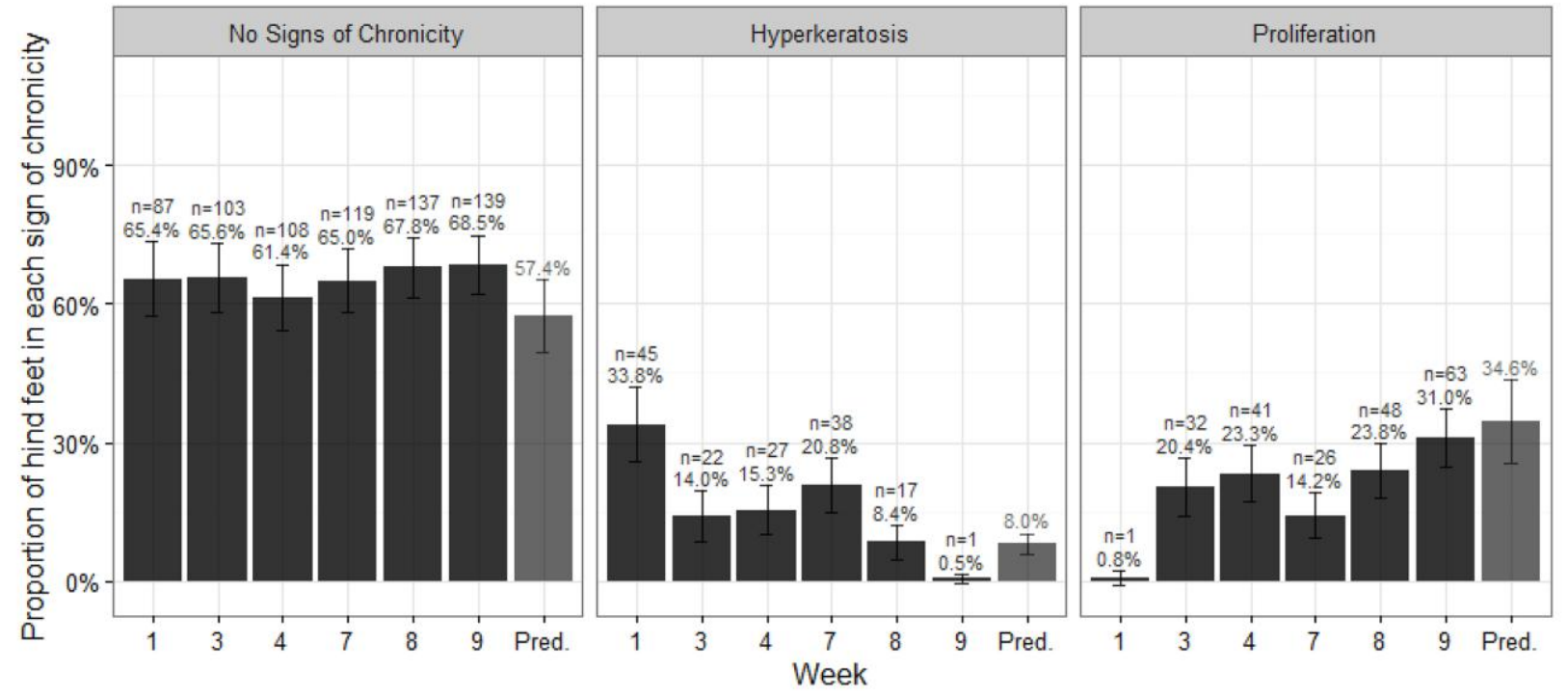




\begin{tabular}{|c|c|c|c|}
\hline Cow ID and Foot & Current M-Stage & Previous M_Stage & Type \\
\hline 7708_LR & 2 & $\theta$ & II \\
\hline 7739_LR & 2 & $\theta$ & II \\
\hline 7747_RR & 2 & & II \\
\hline 7767_RR & 2 & $\theta$ & II \\
\hline 7159_RR & 2 & 2 & III \\
\hline 7167_RR & 2 & 2 & III \\
\hline 7170_LR & 2 & & III \\
\hline 7184_RR & 2 & 3 & III \\
\hline 7196_RR & 2 & 2 & III \\
\hline
\end{tabular}


Table 1: Basic Characteristics of the 4 Sample Data Sets

\begin{tabular}{|c|c|c|c|c|}
\hline & \multicolumn{4}{|c|}{ Data sets } \\
\hline & $\mathrm{A}^{*}$ & B & $\mathrm{C}$ & $\mathrm{D}$ \\
\hline Total observations & 780 & 1,959 & 7,144 & 1,054 \\
\hline Individual cows & 100 & 1,058 & 2,256 & 302 \\
\hline By Feet & yes & no & no & yes \\
\hline Number of unique IDs & 200 & 1,058 & 2,256 & 491 \\
\hline $\begin{array}{l}\text { Number of observations with prev. } \\
\text { M-stage }\end{array}$ & 568 & 696 & 4,307 & 376 \\
\hline Scorer & 6 & 1 & 1 & 1 \\
\hline M-stage & 5 & 2 & 1 & 3 \\
\hline Chronicity & none & yes & yes & yes \\
\hline Pen & yes & yes & yes & yes \\
\hline Type of scoring event & chute & pen walk & pen walk & chute \\
\hline Number of scoring events & 4 & 4 & 5 & 6 \\
\hline Average week interval & 1 & 7.67 & 11.25 & 1.6 \\
\hline
\end{tabular}


Table 2: Transition matrices and probabilities from sequential scoring events per pen

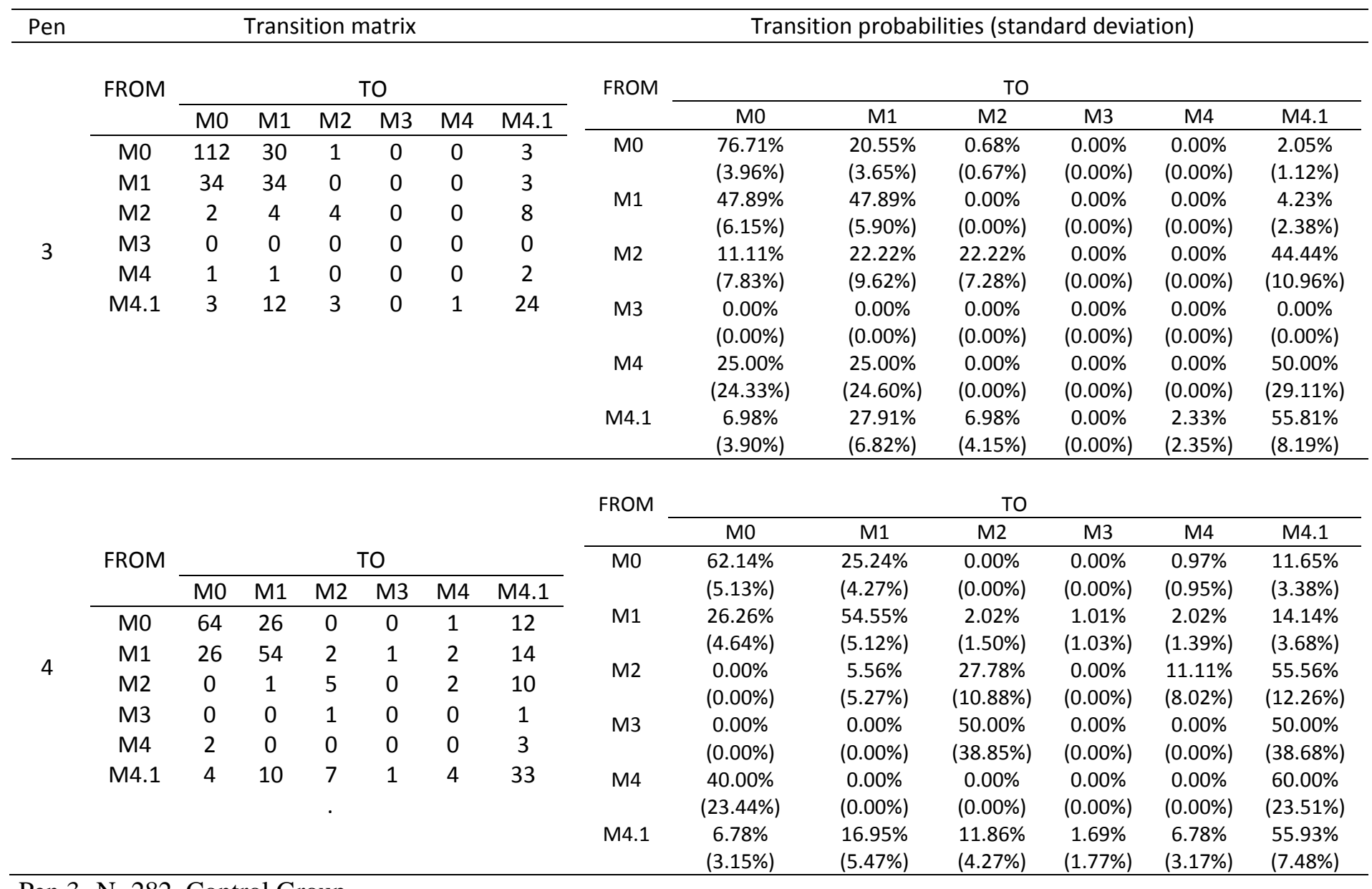

Pen 3- N=282, Control Group

Pen 4- N=286, Treatment Group

Note that rows of transition probabilities add up to $100 \%$. Standard deviation of 1000 bootstraps 